\title{
Optimal Daily Planning for Hydro Power System Coordinated with Wind Power in Areas with Limited Export Capability
}

\author{
J. Matevosyan and L. Söder, Member, IEEE
}

\begin{abstract}
The paper presents a daily planning algorithm for a multi-reservoir hydropower system coordinated with wind power. The planning algorithm applies to the real situation where wind power and hydropower are owned by different utilities, sharing same transmission lines, though, hydropower has a priority for transmission capacity. Coordination is, thus, necessary to minimize wind energy curtailments during the congestion situations. The planning algorithm considers an uncertainty of wind power forecast. Forecast error scenarios are modeled with ARMA series. A scenario reduction algorithm is applied to reduce computational time. Only the planning for the spot market is considered. Thus, once the hydropower production bid is placed on the market it cannot be changed. The solution of the stochastic optimization problem should, therefore, fulfill the transmission constraints for all wind power production scenarios. The developed planning algorithm is applied in a case study. The results are compared to the planning results without coordination.
\end{abstract}

Index Terms - power market, forecasting, optimization, stochastic process, wind power generation, stochastic programming

\section{NOMENCLATURE}

HE Hour equivalent, water flow $1 \mathrm{~m}^{3} / \mathrm{s}$ for 1 hour

Indeces and Index sets

$K \quad$ Indices for all hours in the planning period

$K_{c} \quad$ Indices for congestion hours, $K_{c} \subset K$

$K_{n c} \quad$ Indices for hours without congestion, $K_{n c} \subset K$

$k_{\text {last }}^{\text {day }} \quad$ Last hour in the planning day $k_{\text {last }}^{\text {day }} \in K$

$k_{\text {last }} \quad$ Last hour in the planning period $k_{\text {last }} \in K$

$I \quad$ Indices for hydropower plants (HPPs)

$I_{c} \quad$ Indices for HPPs sharing same transmission capacity with wind farm (WF)

$S_{i} \quad$ Indices for the segments of the HPP production curve, plant $i$

$\Omega_{i} \quad$ Indices for the HPPs directly upstream of plant $i$

$N \quad$ Indices for wind power production scenarios

\section{Parameters}

$\bar{P}_{w}^{n}(k) \quad$ Maximum power production of the WF, hour $k$, scenario $n$ [MW]

The authors would like to acknowledge the financial support for this project from Swedish Energy Agency.

J. Matevosyan and L. Söder are with Royal Institute of Technology (KTH), Stockholm, Sweden. E-mails: julija@ets.kth.se, lennart.soder@ets.kth.se.

\author{
$P_{w}^{a v}$ \\ $\bar{P}_{w}^{h}(k)$ \\ Monthly average wind power production [MW] \\ Maximum power production of the WF, \\ hour $k$, historical data [MW] \\ $\bar{P}_{t}(k) \quad$ Maximum transmission capacity, hour $k$ [MW] \\ $c^{s}(k) \quad$ Forecasted spot price, hour $k$ [SEK/MW] \\ $c \quad$ Price for coordination with WF [SEK/MW] \\ $\bar{u}_{i s} \quad$ Maximum discharge in plant $i$, segment $s[\mathrm{HE}]$ \\ $\mu_{i s} \quad$ Prod. equiv. for plant $i$, segment $s$ [MWh/HE] \\ $D_{k} \quad$ Local load, hour $k$ [MW] \\ $\bar{x}_{i} \quad$ Maximum reservoir content [HE] \\ $x_{i}^{o} \quad$ Initial reservoir content [HE] \\ $x_{i}^{\text {last }}$ \\ $w_{i}(k)$ \\ Reservoir content at the end of the period [HE] \\ $u_{i s}(k)$ \\ $y_{i}$ \\ Water inflow to the reservoir $i$, hour $k$ [HE] \\ Water discharge from plant $i$, segment $s$, hour $k$ [HE] \\ Water spillage from plant $i$, hour $k$ [HE] \\ $\tau_{i j} \quad$ Water delay time from reservoir $i$ to the \\ next downstream reservoir $j$ \\ $H_{i} \quad$ Number of whole hours from reservoir $i$ to the \\ next downstream reservoir \\ $M_{i} \quad$ Number of minutes additional to the whole hours \\ from reservoir $i$ to the next downstream reservoir \\ $P_{i}(k) \quad$ HPP production in stations $I_{c}$ without coordination
}

Variables

$\Delta P_{i s}(k)$ Power saved in plant $i$, segment $s$, hour $k$ [MW]

$\Delta u_{i s}(k)$ Increase in discharge of hydro plant $i$, segment $s$, hour $k$ [MW]

$x_{i}(k) \quad$ Reservoir content at the hydro plant $i$, hour $k$ [HE]

$z^{b} \quad$ HPPs income, base case planning [SEK]

$z^{c} \quad$ HPPs additional income, coord. strategy [SEK]

$z^{\text {tot }} \quad$ HPPs total planned income[SEK]

\section{INTRODUCTION}

$\mathbf{T}$ HE best conditions for integration of wind power are in remote areas free of obstacles. However, the transmission system in such areas might not be fully dimensioned to accommodate additional large-scale power plants. The existing power plants in the same area might have transmission capacity already reserved for their production.

When power transmission is congested it could be possible to store excess wind energy. The existing conventional power plants with possibilities of fast production control and sufficient storage capability (e.g. hydro power plants or gas power plants) could be used for this purpose. 
The coordination of wind power and hydro power has been studied earlier in connection with several different problems. In [1] this option is considered in generation expansion planning, where two investment possibilities are compared: new hydro power plant (HPP) vs new wind farm (WF). In [2], [3] it is analyzed how wind power would effect the market price. The research in [2], [3] is aimed at hydro power utilities that consider investments in wind power. In [4] technical advantages of hydro and wind power coordination are analyzed. In [5] several coordination strategies were suggested assuming separate ownership of wind power and hydropower. The strategies are then evaluated using historical data of hydro power operation and wind speed measurements from the studied site. None of these papers, however, treat hydro power production planning for daily operation, considering the coordination with wind power.

In [6] the dynamic programming algorithm is presented for daily planning of coordinated operation of wind parks and generic energy storage. In [7] optimization problem is formulated for daily planning for wind parks and pumped storage hydro power plants is developed. In both papers, however, it is assumed that storage is owned by the wind power utility, a multi-reservoir hydro power system is also not considered for this application.

In this paper the daily planning algorithm is developed for a conventional multi-reservoir hydro power system in coordination with a wind farm, according to one of the coordination strategies developed in [5]. Wind power and hydro power are assumed to be owned by different utilities. It is assumed that the wind farm and the HPP system are sharing the same transmission line(s), but the hydro power utility is assumed to have a priority for the transmission capacity. Thus, coordination is necessary in order to minimize wind energy curtailments during the congestion situations. The wind power utility is assumed to be paying the hydro power utility for the coordination service. The purpose of this planning is to provide the hydro power utility with optimal hourly bids to the dayahead market (spot market), considering an uncertainty of the wind power forecast. The planning problem is formulated as a stochastic optimization program.

Furthermore, an algorithm is also elaborated to evaluate how the developed coordinated daily planning would affect the operation of the HPP system and the WF in the long run. The purpose of this algorithm is to run the coordinated daily planning successively day by day over a certain period of time (e.g. one year). The results of the coordinated planning could then be compared to the results of the uncoordinated planning for the same period in order to evaluate the impact of purposed coordination on hydro power and wind power utilities.

The paper is outlined as follows. In Section II the flow chart for the hydro power planning in coordination with wind power is presented and explained step by step in the respective subsections. In Section III the algorithm for evaluation of the coordinated planning is described. Finally, both shortterm planning and evaluation algorithms, developed in this paper, are applied in a case study in Section IV. Section V summarizes the main conclusions and plans for the future work.

\section{COORDinAtion STRATEGY WITH WIND POWER}

In this paper hydro power production planning is coordinated with wind power in the following way. For each hour of the coming day, if transmission congestion is expected, the hydro power utility could decrease its planned production, considering the constraints of the hydro reservoirs. Hydro power is, thus, stored in reservoirs and wind farm can use available transmission capacity. Each MW of stored hydro power corresponds to $1 \mathrm{MW}$ of transmission capacity that is, thus, made available for excess wind energy. Stored hydro power could be used in the hours without congestion. The wind power utility is assumed to pay to the hydro power utility for this service. Therefore, in the coordinated planning it is important to keep track of the exact amount of hydro power production reductions due to wind power and transmission congestions. For this purpose the planning is divided into two stages:

1) base case hydro power planning without consideration of wind power;

2) re-planning of hydro power production, considering wind power forecast and its uncertainty.

The following assumptions are made for both stages of the planning:

- The spot prices are deterministic (spot price forecast may be used);

- The planning is based on the price taking assumption;

- Only the planning for the spot market is considered;

- Hydro power planning for the coming day is assumed to be performed at $11 \mathrm{pm}$ the day before, in order to place the bids on the spot market that closes at $12 \mathrm{pm}$;

- Beside the coming day the rest of the ongoing week is also included in the planning. This is done in order to keep hydro reservoir content at the end of the week fixed in accordance with mid-term planning [9] and to account in the re-planning stage for the wind power that might be produced during the following days. However, only the planning results for the coming day are used for bidding on the spot market.

The planning is performed for each day in accordance with the short-term HPP production planning part of the flow chart, Fig. 1. This part of the flow chart is discussed step by step in the following subsections.

\section{A. Initial data}

For the day of the planning corresponding input data are loaded (block 1): water inflow, the initial reservoir content and the final reservoir content at the end of the ongoing week, the spillage and the discharge in the preceding hours to account for the water delay time between the reservoirs.

\section{B. Hydro Power Planning, Base Case}

This subsection discusses modeling details of the base case hydro power planning without consideration of wind power, (block 2), Fig. 1.

Generally, hydropower production is planned differently by different utilities. There is a large variation in modeling regarding the level of details, representation of uncertainties etc. 
Furthermore, the strategies are not always optimal, because of unexpected events, e.g. generator outages or participation in the regulating market etc.

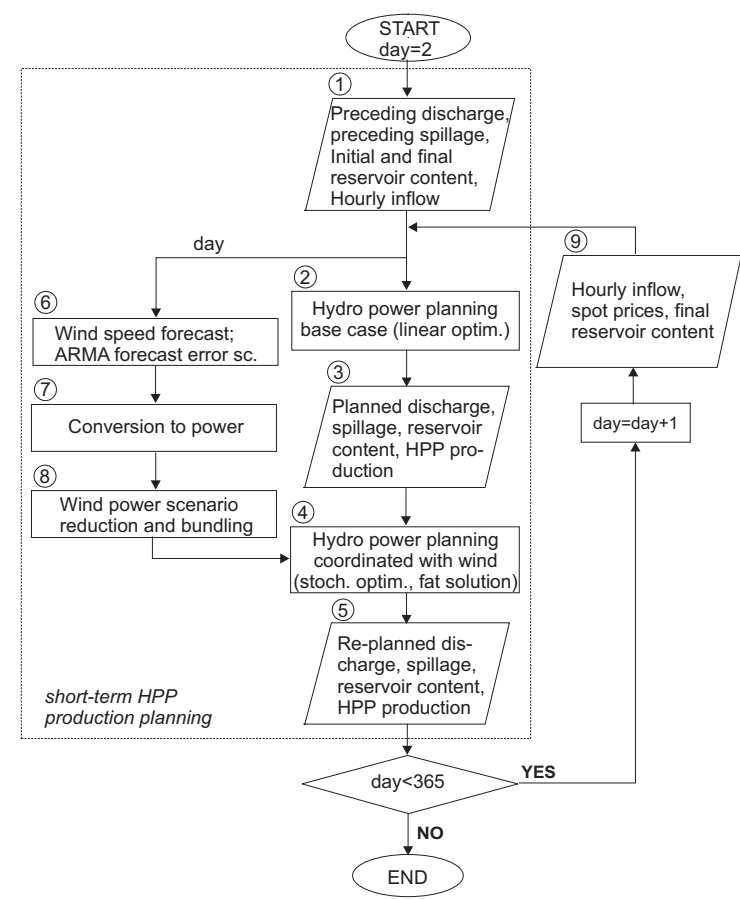

Fig. 1. Flow chart of the successive planning for one year

A hydro power production characteristic of each unit in a hydro power plant is a family of nonlinear functions of the respective reservoir head, efficiency of the turbine/generator set and water discharge. The generation functions of all production units form a family of nonlinear and nonconcave production curves each for a specified value of the head [10]. In most planning methods the head dependence is neglected to avoid nonlinearities, which allows using a single production curve. In this paper for simplicity this single production curve is further approximated by a concave piecewise linear function, [11]. The local best efficiency points of the true generation function and the point of maximum discharge are used as the breakpoints, Fig. 2, The slope of each linear segment is called the production equivalent, $\mu_{i s}$. This simplification leads to a linear optimization problem. For other methods for modeling production curve see e.g. [12] and [10].

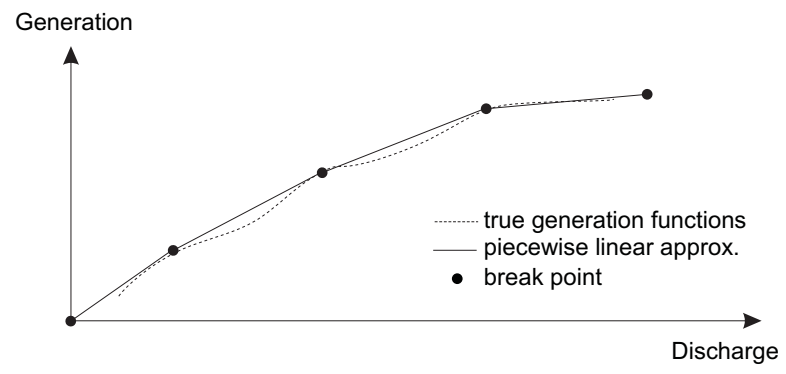

Fig. 2. Production curve of the hydro power plant.

The planning algorithm presented in this paper is structured so that more detailed representation of the production characteristic could be introduced, if necessary.

The objective function of the hydro power producer is to maximize the income from produced power:

$$
\max z^{b}=\sum_{k \in K} c^{s}(k) \sum_{i \in I} \sum_{s \in S_{i}} u_{i s}(k) \mu_{i s}
$$

where $u_{i s}(k)$ is water discharge at hour $k$, at power plant $i$, in segment $s$ of the production function, $c^{s}(k)$ is a spot price at hour $k, \sum_{i \in I} \sum_{s \in S_{i}} u_{i s}(k) \mu_{i s}$ is, thus, power that hydro power producer sells on the spot market for hour $k$.

The hydrological constraints describe couplings between adjacent hydro power plants in the multi-reservoir hydro system.

$$
\begin{aligned}
& x_{i}(k+1)=x_{i}(k)-\sum_{s \in S_{i}} u_{i s}(k)-y_{i}(k)+w_{i}(k) \\
& +\sum_{j \in \Omega_{i}}\left[\sum_{s \in S_{j}} u_{j s}\left(k-\tau_{i j}\right)+y_{j}\left(k-\tau_{i j}\right)\right], \\
& \forall \quad i \in I, \forall k \in K
\end{aligned}
$$

where $x_{i}(k+1), u_{i s}(k), u_{j s}(k), y_{i}(k), y_{j}(k), x_{i}(k)$ are variables, $w_{i}(k)$ is a parameter.

The reservoir content of the particular HPP is affected by water spillage and discharge in HPPs directly upstream. The delay time $\tau_{i j}$ between HPP $j$ and HPP $i$ directly downstream is assumed to be defined in $H_{j}$ hours and $M_{j}$ minutes. The discharge considering the delay time is then expressed as:

$$
\begin{aligned}
& u_{j s}\left(k-\tau_{j i}\right)=\frac{M_{j}}{60} u_{j s}\left(k-H_{j}-1\right) \\
& +\frac{60-M_{j}}{60} u_{j s}\left(k-H_{j}\right), \forall j \in \Omega_{i}, s \in S_{j}, k \in K
\end{aligned}
$$

Analogously, the spillage considering the delay time can be determined [8].

Power transmission from the studied site is limited, thus:

$$
\sum_{i \in I_{c}} \sum_{s \in S_{i}} u_{i s}(k) \mu_{i s}-D(k) \leq \bar{P}_{t}, \quad \forall k \in K
$$

where $D(k)$ is hourly load in the studied site and $\bar{P}_{t}$ is power transmission limit from the studied site.

There are also reservoir content, discharge and spillage limitations:

$$
\begin{gathered}
0 \leq x_{i}(k) \leq \bar{x}_{i}, \quad \forall i \in I, \forall k \in K \\
0 \leq u_{i s}(k) \leq \bar{u}_{i s}, \quad \forall i \in I, \forall s \in S_{i}, \forall k \in K \\
0 \leq y_{i}(k) \leq \bar{y}_{i}, \quad \forall i \in I, \forall k \in K
\end{gathered}
$$

Initial reservoir content is assumed known:

$$
x_{i}(0)=x_{i}^{o}
$$

The reservoir content at the end of the planning period is fixed in accordance with mid-term production planning:

$$
x_{i}\left(k_{\text {last }}\right)=x_{i}^{\text {last }}
$$

The results of the base case hydro power planning: planned water discharge, HPP production, spillage, reservoir content (block 3) - are passed as parameters to the re-planning program that includes coordination with wind power (block 4). 


\section{Wind Power ARMA Forecast Error Scenarios}

This subsection discusses blocks 6-8 of the flow chart, Fig. 1. These blocks prepare the input data for the re-planning program (block 4).

A wind speed forecast for the planning day is obtained from e.g. numerical meteorological programs. A wind speed forecast is never perfect and the forecast error should be considered.

A model for wind speed forecast error (block 6) is developed in [14]. It is assumed that data concerning accuracy of the forecast are known. The model then simulates possible outcomes (scenarios) of the wind speed forecast errors that have the correct statistical behavior. The model is based on Auto Regressive Moving Average series (ARMA), [13], defined as:

$$
\begin{aligned}
& \Delta V(0)=0 \\
& Z(0)=0 \\
& \Delta V(k)=\alpha \Delta V(k-1)+Z(k)+\beta Z(k-1)
\end{aligned}
$$

where $\Delta V(k)$ is wind speed forecast error in $k$-hour forecast, $Z(k)$ is random Gaussian variable with standard deviation $\sigma_{Z}$, $\alpha$ and $\beta$ are parameters.

The wind speed scenario for each hour, $v^{n}(k)$, can then be calculated as the sum of the wind speed forecast, $v^{f}(k)$ and the wind speed forecast error scenario, $\Delta v^{n}(k)$, i.e. the outcome of $\Delta V(k)$ :

$$
v^{n}(k)=v^{f}(k)+\Delta v^{n}(k), \forall n \in N
$$

where $N$ is number of forecast error scenarios.

The parameters $\sigma_{Z}, \alpha$ and $\beta$ are identified using the least square fitting, minimizing a difference between sample forecast error variance, based on data from the site, and modeled forecast error variance, see [14] for details.

Obtained wind speed scenarios are then converted to wind power production scenarios (block 7), $\bar{P}_{w}^{n}(k)$, using power curves of the respective turbines, [15].

The wind power scenario model is only applied for the planning day. For the remaining days, until the end of the ongoing week, included in the planning, monthly average wind power production $P_{w}^{a v}$ is used instead of wind power scenarios $\bar{P}_{w}^{n}(k)^{1}$, Fig. 3. It is calculated using power curve from the monthly average wind speed according to statistical data.

\section{Scenario reduction and bundling}

Scenarios generated as described in the previous section form the scenario fan. To assure that stochastic properties of the process are represented correctly many scenarios should be generated.

The computational effort for solving scenario-based optimization models depends on the number of scenarios. Therefore it is necessary to reduce the original scenario fan so that it would have a smaller number of scenarios but stochastic properties would not be changed more than necessary compared to the original scenario fan.

\footnotetext{
${ }^{1}$ In the following subsections the equations are written in general form however, for all $k>K_{\text {last }}^{\text {day }}, \bar{P}_{w}^{n}(k)$ should be substituted by $P_{w}^{a v}$
}

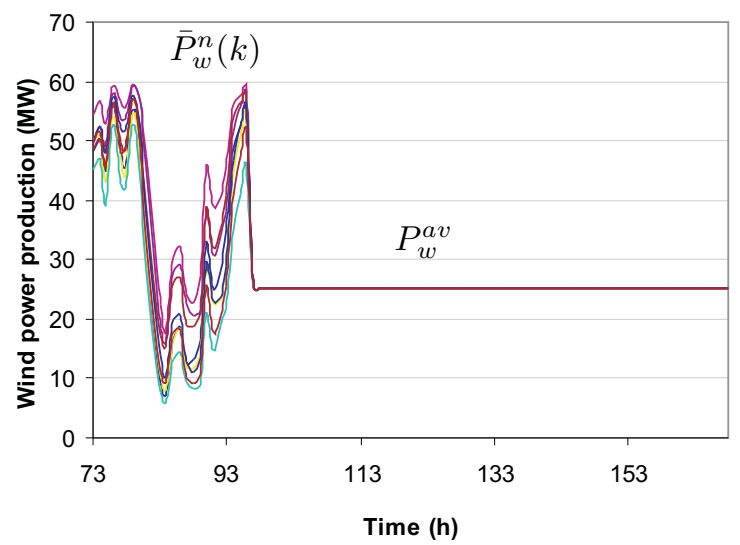

Fig. 3. Example of wind power production scenarios for $60 \mathrm{MW}$ wind farm for the planning day (Thursday in this example) and for the rest of the ongoing week included in planning

The scenario reduction approach applied here (block 8) is presented in details in [16] and [17]. The scenario reduction algorithm reduces and bundles the scenarios using the Kantorovich metric, which assures that as many scenarios as possible are reduced without violating the given tolerance criteria, Fig. 4.

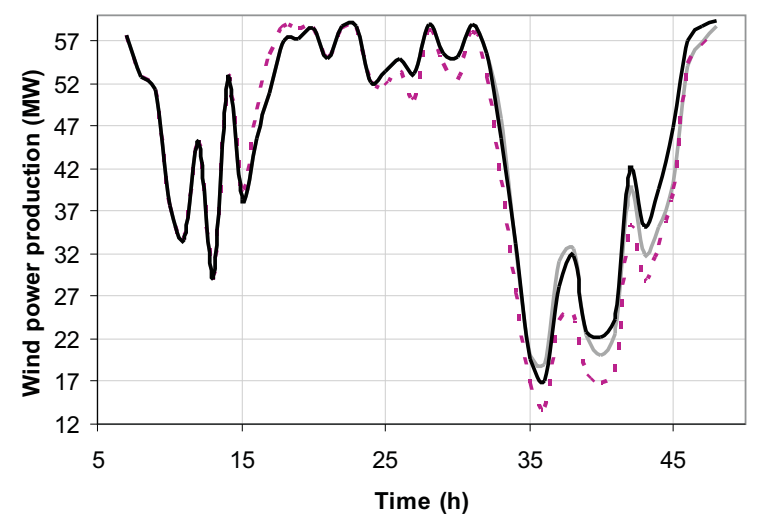

Fig. 4. Example of the reduced scenario tree, for $60 \mathrm{MW}$ wind farm for the planning day

\section{E. Hydro power re-planning coordinated with wind power}

In the coordinated planning (block 4), the base case hydro power production plan (from block 2-3) is adjusted to account for the wind power.

If, according to the base case hydro power production plan (block 3) and wind power production scenarios (block 8), power transmission would be congested, then planned HPP production could be decreased, in order to allow the WF to use transmission capacity. The expected hours with congestion are, thus, defined as follows:

$$
\text { if } \begin{array}{r}
\sum_{i \in I_{c}} \sum_{s \in S_{i}} \mu_{i s} u_{i s}(k)+\bar{P}_{w}^{n}(k)-D(k)>\bar{P}_{t},=> \\
k \in K_{c}, \\
\forall n \in N
\end{array}
$$

Stored hydro power could be used later, during the hours without congestion. 
The expected hours without congestion are defined as:

$$
\begin{aligned}
& \text { if } \sum_{i \in I_{c}} \sum_{s \in S_{i}} \mu_{i s} u_{i s}(k)+\bar{P}_{w}^{n}(k)-D(k) \leq \bar{P}_{t},=>k \in K_{n c} \text {, } \\
& \forall n \in N
\end{aligned}
$$

where $\sum_{i \in I_{c}} \sum_{s \in S_{i}} \mu_{i s} u_{i s}(k)$ is planned hydro power production in plant $i$, hour $k$ according to the base case, $\bar{P}_{w}^{n}(k)$ is wind power production scenario $n$ for hour $k, \bar{P}_{t}$ is available transmission capacity.

In the coordinated planning two new variables are introduced: additional discharge $\Delta u_{i s}(k)$ and power production decrease $\Delta P_{i s}(k)$. The new variables make it possible to track changes in the coordinated production plan compared to the base case.

Here the objective of the hydro power producer is to maximize the income from coordination with wind power:

$$
\begin{aligned}
\max z^{c} & =\sum_{k \in K}\left(c^{s}(k)\left(\sum_{i \in I} \sum_{s \in S_{i}}\left(\Delta u_{i s}(k) \mu_{i s}-\Delta P_{i s}(k)\right)\right)\right. \\
& \left.+\sum_{i \in I_{c}} \sum_{s \in S_{i}} c \cdot \Delta P_{i s}(k)\right)
\end{aligned}
$$

The hydro power producer is assumed to be paid for production reduction at the stations $I_{c}$, as this relaxes congestion of power transmission and allows the WF to produce energy, that otherwise would be curtailed. The price $c$, SEK/MWh, is estimated as yearly average loss due to wind energy curtailments, based on historical data:

$$
c<\frac{\sum_{k \in K_{c}} c^{s}(k)\left(\sum_{i \in I_{c}} P_{i}(k)+\bar{P}_{w}^{h}(k)-P_{12}\right)}{\sum_{k \in K_{c}}\left(\sum_{i \in I_{c}} P_{i}(k)+\bar{P}_{w}^{h}(k)-P_{12}\right)}
$$

where $P_{i}(k)$ is HPP production in stations $I_{c}$ without coordination, $\bar{P}_{w}(k)^{h}$ is wind power production calculated from historical wind speed data, $c^{s}(k)$ is a spot price for the same period in the past. The numerator corresponds to the economical losses due to wind energy curtailments according to historical data and denominator corresponds to total wind energy curtailments in that period. Thus, $c$ is the upper limit for the price that the wind power utility is prepared to pay to the hydro power utility for the coordination.

The hydrological constraints (2) need to be adjusted as follows:

$$
\begin{aligned}
& x_{i}(k+1)=x_{i}(k)-\sum_{s \in S_{i}} u_{i s}(k)-y_{i}(k)+w_{i}(k) \\
& +\sum_{j \in \Omega_{i}}\left[\sum_{s \in S_{j}} u_{j s}\left(k-\tau_{i j}\right)+y_{j}\left(k-\tau_{i j}\right)\right] \\
& +\sum_{s \in S_{i}}\left(\frac{\Delta P_{i s}(k)}{\mu_{i s}}-\Delta u_{i s}(k)\right) \\
& +\sum_{j \in \Omega_{i}} \sum_{s \in S_{i}}\left(-\frac{\Delta P_{j s}\left(k-\tau_{j i}\right)}{\mu_{j s}}+\Delta u_{j s}\left(k-\tau_{j i}\right)\right), \\
& \forall i \in I, \forall k \in K
\end{aligned}
$$

Here discharges $u_{i s}(k), u_{j s}(k)$, spillage $y_{i}(k), y_{j}(k)$ are already known parameters calculated in the base case planning (blocks 2-3), other quantities are variable. The last two terms (in the large parentheses) include the effect on the hydro reservoir content from the hydro production reduction $\frac{\Delta P_{i s}(k)}{\mu_{i s}}$ and from the disposal of stored water $\Delta u_{i s}(k)$, in the local station and the stations directly upstream. The HPP production reduction, $\Delta P_{i}(k)$ is in $\mathrm{MW}$. However the reservoir content in the hydrological constraint is expressed in hour equivalents $\mathrm{HE}$, thus, $\Delta P_{i}(k)$, is converted to corresponding decrease of discharge in HE, using HPP's production function, Fig. 2.

The decrease of discharge should not exceed planned discharge $u_{i s}(k)$, according to the base case:

$$
0 \leq \frac{\Delta P_{i s}(k)}{\mu_{i s}} \leq u_{i s}(k), \forall i \in I, \forall s \in S_{i}, \forall k \in K
$$

Water discharge should always be within the limits set by technical and environmental constraints, i.e.:

$$
0 \leq u_{i s}(k)+\Delta u_{i s}(k) \leq \bar{u}_{i s}, \quad \forall i \in I, \forall s \in S_{i}, \forall k \in K
$$

Additional water spillage due to energy storage in hydro reservoirs is not allowed in the coordination strategy. It seems meaningless to integrate wind power if it results in energy spillage at other power plants.

Hydropower production at stations $I_{c}$ should only be reduced during the congestion situations in order to allow wind power production without or with lower energy curtailment:

$$
\begin{aligned}
& \sum_{i \in I_{c}} \sum_{s \in S_{i}} \Delta P_{i s}(k) \leq \sum_{i \in I_{c}} \sum_{s \in S_{i}} \mu_{i s} u_{i s}(k)+\bar{P}_{w}^{n}(k)-D(k) \\
& -\bar{P}_{t}, \forall k \in K_{c}, \forall n \in N
\end{aligned}
$$

Constraint (17) states that stored wind energy should be less than or equal to potential wind energy curtailment during the congestion situation. And, on the contrary, when power transmission is not congested, additional hydro power production should not exceed transmission margin:

$$
\begin{aligned}
& \sum_{i \in I} \sum_{s \in S_{i}} \Delta u_{i s}(k) \mu_{i s} \leq \bar{P}_{t}+D(k)-\sum_{i \in I} \sum_{s \in S_{i}} \mu_{i s} u_{i s}(k) \\
& -\bar{P}_{w}^{n}(k), \forall k \in K_{n c}, \forall n \in N
\end{aligned}
$$

If transmission is not congested, no energy is stored in hydro reservoirs of the $I_{c}$ stations:

$$
\sum_{i \in I_{c}} \sum_{s \in S_{i}} \Delta P_{i s}(k)=0, \quad \forall k \in K_{n c}
$$

Otherwise, if transmission is congested there should be no additional hydropower production in the $I_{c}$ stations, i.e.:

$$
\sum_{i \in I_{c}} \sum_{s \in S_{i}} \Delta u_{i s}(k)=0, \quad \forall k \in K_{c}
$$

Hydro reservoir content at the beginning of the planning period is assumed known and at the end of the period is fixed in accordance with the mid-term planning, (8) and (9). Reservoir content should always be within the limits, set by technical and environmental constraints ,(5).

In this paper only the spot market is considered, consequently, the production plan once submitted to the spot market cannot be changed during the day. This means that the solution of the optimization problem should satisfy all constraints at 
any outcome of wind power production scenarios. A production program like this is called "fat solution" [18].

The results of the optimization (block 4): re-planned water discharge, adjusted HPP production, water spillage, reservoir content at the end of the day (block 5).

The total bid of the HPP producer to the spot market for each hour $k$ is calculated as the sum of the base case production and additional production due to coordination with wind power, i.e.:

$$
\left.\sum_{i \in I} \sum_{s \in S_{i}}\left(\left(u_{i s}(k)+\Delta u_{i s}(k)\right) \mu_{i s}-\Delta P_{i s}(k)\right)\right)
$$

The total planned income of the HPP producer from the coordination strategy is calculated as a sum of the base case income (block 2) and additional income from coordination (block 4), i.e.:

$$
z^{t o t}=z^{b}+z^{c}
$$

\section{EVALUATION OF THE PLANNING ALGORITHM}

It is necessary to evaluate how the developed planning algorithm will impact the operation of the HPP system and the WF. The work flow developed for this purpose, Fig. 1, allows to run the daily planning successively for the certain period of time (e.g. one year). It is convenient to choose a period in the past, thus, all necessary input data (spot prices, water inflows, wind speeds) would be available.

The planning is done as presented in Section II for each day. The results of the optimization: re-planned water discharge, adjusted HPP production, water spillage, reservoir content at the end of the day (block 5) - are used as input data for the next day planning. The cycle continues until the end of the evaluation period.

In order to run the uncoordinated planning for the same period of time, same work flow, Fig. 1, excluding the blocks 6-8 and 4-5 is used. The results of the successive planning for both cases (with and without coordination) could then be compared.

To evaluate the impact of coordination on the wind power utility WF production and income of the utility are calculated for the same period as above, for the cases without and with coordination. In latter case, as described in Section II, wind power utility is assumed to pay hydro power utility for each MW of transmission capacity that is made available by means of coordination. In both cases wind energy is assumed to be curtailed if the congestion in the transmission occurs.

\section{CAse Study}

The developed planning strategy is tested in a case study. The case study is based on the actual case where the Swedish company is interested to build a WF in the mountainous area in the northern Sweden near the Norwegian border. The amount of planned wind power installation is 30 to $90 \mathrm{MW}$. The wind conditions are very good in this area but transmission capacity of the lines is limited to $350 \mathrm{MW}$. On the Swedish side 250 MW are reserved for hydro power production (5 HPP stations on the Ume river) and the rest, $100 \mathrm{MW}$, for power exchange with Norway. Although the power line is not always utilized to $100 \%$ the connection of WF has been rejected.
In the case study 9 stations of the Ume river are modelled, Fig. 5, and production of the 5 upper stations (total installed capacity $250 \mathrm{MW}$ ) are assumed to be coordinated with $60 \mathrm{MW}$ of wind power. These stations and the wind farm are sharing $250 \mathrm{MW}$ of transmission capacity.

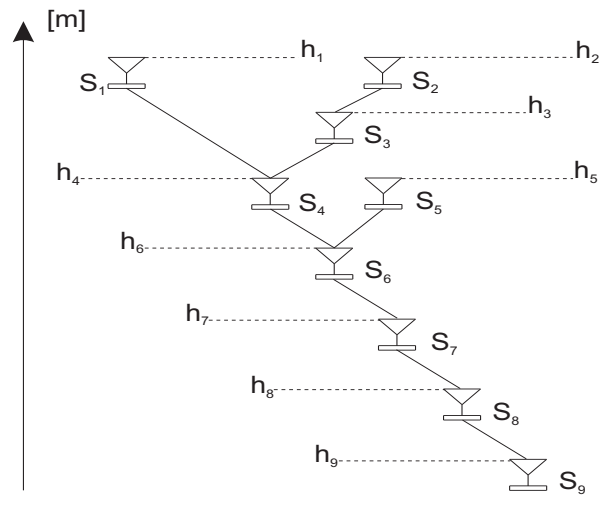

Fig. 5. Studied HPP system of Ume river

In the case study the uncoordinated hydro power planning and coordinated with wind power is done successively day by day for one year, Fig. 1. For apt illustration the results for one week are shown bellow. The following assumptions are made:

- It is assumed that there is no local load in the area with congestion problems.

- Spot prices and hourly inflow to hydro reservoir system of Ume river from 2001 are used as input data.

- Reservoir contents of the HPP system at the end of each week are fixed to the respective values from 2001.

- Wind speed forecast data were not available and thus actual wind speeds from the studied site from 2001 are used as wind speed forecast.

- Forecast error is modeled as described in Section II-C. Forecast error data from Eastern Denmark from 2003 are used to calculate parameters for ARMA series.

- 1000 forecast error scenarios is first generated, then the initial scenario fan is reduced by $80 \%$ and then additionally by $1 \%$ per time step in the scenario reduction and bundling process, see Section II-D.

- Power curve of $2 \mathrm{MW}$ wind turbine Vestas80 is used to convert wind speeds to power, wind farm smoothing effect is not considered.

Fig. 6 shows planned hydro power production for the first five stations of the studied HPP system. The results are shown for uncoordinated planning and for the planning coordinated with wind power for the first week in January.

According to the uncoordinated planning in some hours hydro power production at the first five stations of the HPP system is as high as available transmission capacity. In the coordinated planning hydro power production at these stations is reduced to free some transmission capacity for expected wind power. Reservoir content of the HPP stations at the end of the week is fixed in accordance to data from 2001. This means that coordination with wind power effects HPP system only within one week. 


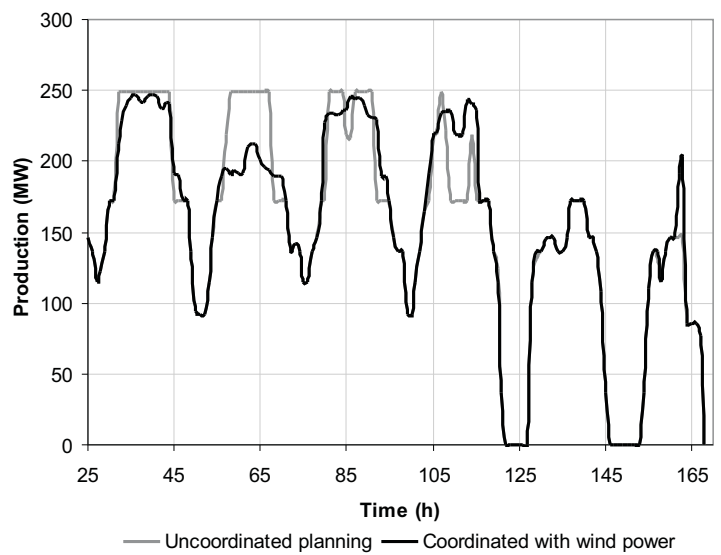

Fig. 6. Planned hydro power production for the uncoordinated planning and coordinated planning for the stations 1-5 of the studied HPP system

Figure 7 and Figure 8 show reservoir content and hourly discharge at the third hydro power plant for the uncoordinated and coordinated planning. The reservoir content at the end of the week is fixed in accordance to data from 2001. This means that the coordination affects the HPP system only within one week.

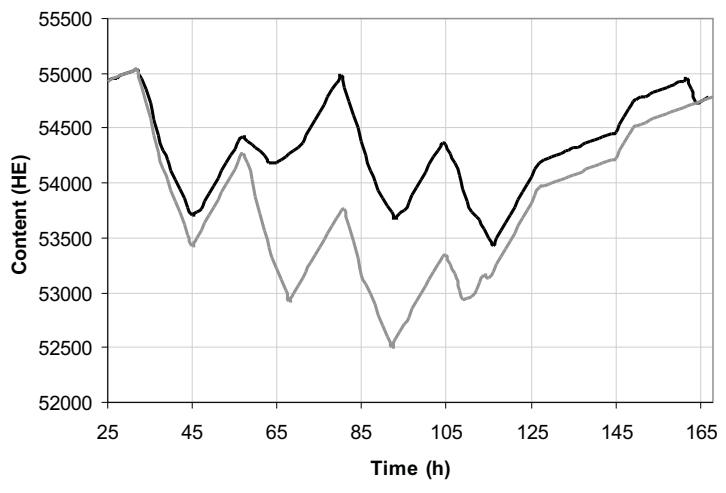

— Coordinated with wind power — Uncoordinated planning

Fig. 7. Reservoir content at the third hydro power plant for the uncoordinated planning and coordinated planning

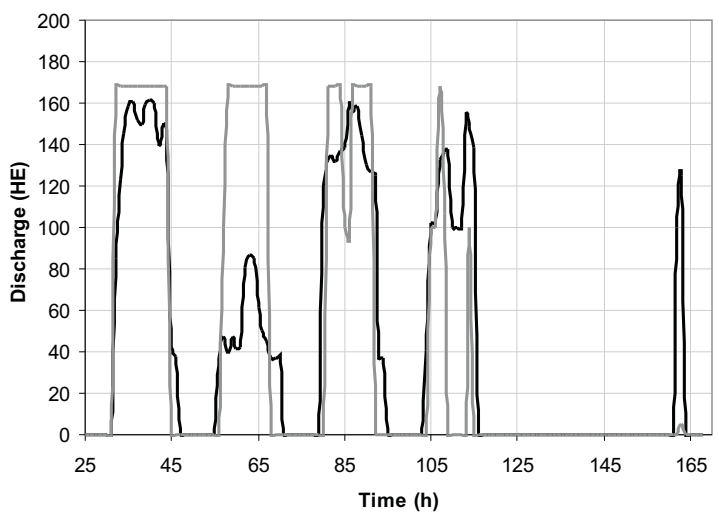

— Coordination of wind and hydro _- Uncoordinated planning

Fig. 8. Hourly discharge at the third hydro power plant for the uncoordinated planning and coordinated planning
Planned wind power production is calculated from the wind power forecast considering the transmission margin left after hydro power production is scheduled. This is due to assumption that hydro power system has a priority to transmission capacity from the studied area. Fig. 9 shows planned wind power production according to the uncoordinated planning and coordinated planning.

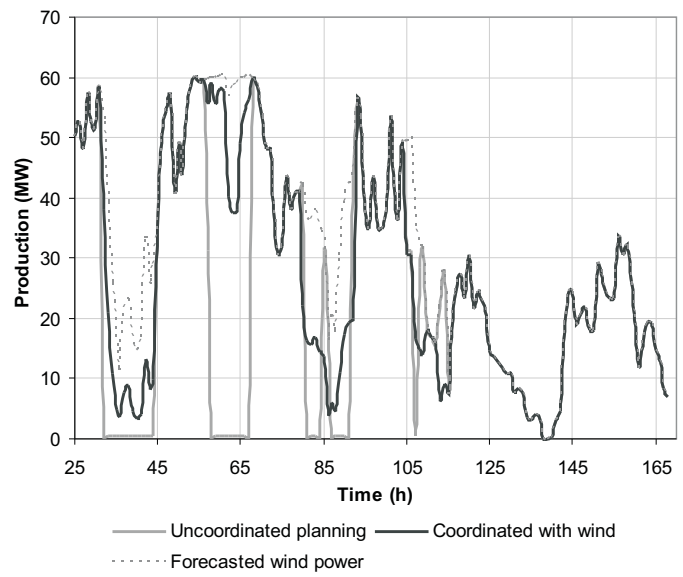

Fig. 9. Planned wind power production with and without coordination, and wind power production forecast

Wind energy curtailment is reduced during the studied week from $1312 \mathrm{MWh}$ in the uncoordinated case to $659 \mathrm{MWh}$ in the coordinated case, i.e. by about $50 \%$. This reduction by $50 \%$ is also observed for the whole simulated period. Some wind energy curtailment still prevails due to technical limitations of the considered HPP system.

Fig. 10 shows power transmission for the studied week. In the coordinated case power transmission is higher than in the case when wind power and hydro power are not coordinated.

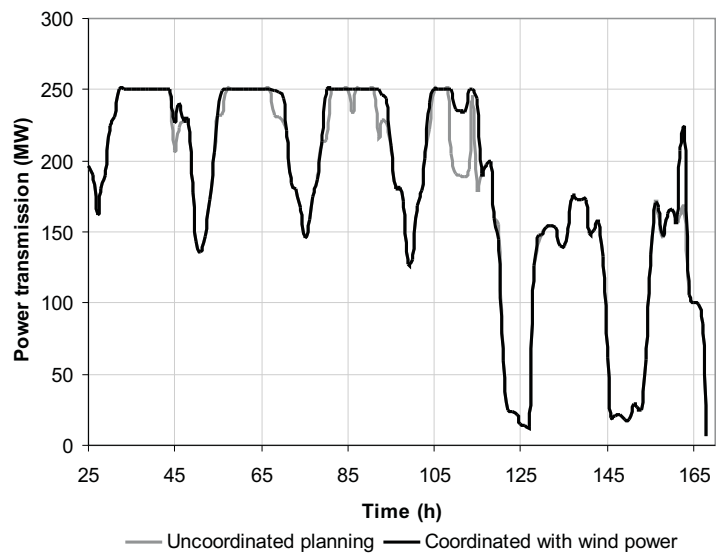

Fig. 10. Power transmission with and without coordination

Fig. 11 illustrates a difference in daily income of 9 HPP stations of Ume river between the coordinated planning and uncoordinated planning for one month. The difference is sometimes negative (i.e. the income planned according to the uncoordinated case is higher than the income planned according to the coordinated case). This is due to the monthly average wind power assumption after the planning day till the end of 
the ongoing week, Fig. 3, on the one hand and fixed reservoir content constraint at the end of the week (9) on the other hand. For the whole year the difference in income between the coordinated planning and the uncoordinated planning is $5.5 \cdot 10^{6} \mathrm{SEK} \approx 0.59 \cdot 10^{6} \mathrm{EUR}$.

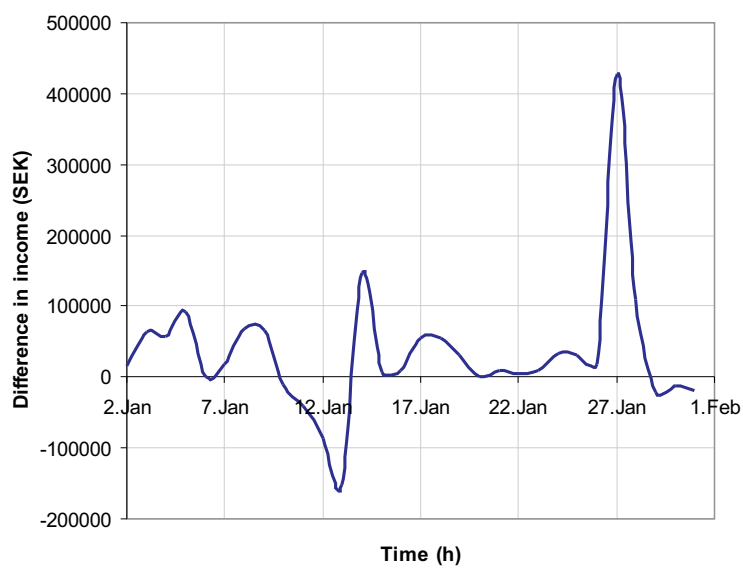

Fig. 11. Difference in daily income of 9 HPP stations of Ume river and for $60 \mathrm{MW}$ WF for the uncoordinated and coordinated planning, January

The planned income of the wind farm owner in the uncoordinated case and in the case with coordination is almost the same, due to the fact that maximum price for coordination service (13), is applied in this case study. In practice this price should be based on agreement between HPP and WF owners and should be less than the maximal value.

\section{CONCLUSIONS AND FUTURE WORK}

This paper has presented the planning strategy for coordination of wind power and hydro power in areas with congestion problems. The planning strategy takes the uncertainty of wind power forecast into account. The planning strategy was tested in the case study, which has shown that coordination of wind power and hydro power can be mutually beneficial for the wind farm and hydro power owner. The coordination decreases wind energy curtailments and also leads to more efficient utilization of the existing transmission lines.

In this paper wind power forecast was used for the planning day and monthly average wind power production was assumed till the end of the current week. This negatively effects the results of the planning and, thus, more detailed model should be developed in future.

The "fat solution" to the stochastic optimization problem usually rather expensive, i.e. it would lead to unnecessary wind energy curtailments. In the future the possibility to trade on the regulating market will be also included in the optimization problem, to calculate optimal hydro power production adjustments during the day.

\section{REFERENCES}

[1] S. Krau, G. Lafrance and L. Lafond, "Large scale wind farm integration: a comparison with a traditional hydro option", in Proc. 2002 EWEA Global Wind Power Conference.
[2] K.-O. Vogstad, "Utilizing the complementary characteristics of wind power and hydropower through coordinated hydro production scheduling using EMPS model", in Proc. 2000 Nordic Wind Energy Conference, Trondheim. [Online]. Available: www.stud.ntnu.no/ klausv

[3] K.-O. Vogstad, H. Holttinen, A. Botterud and J. O. G. Tande, "System benefits of coordinating wind power and hydropower in a deregulated market", in Proc. 2000 Wind Power for the 21st century. [Online]. Available: www.stud.ntnu.no/ klausv

[4] J. O. G. Tande, K. Uhlen and T. Gjengedal,"Options for Large Scale Integration of Wind Power", in Proc. 2005 IEEE PowerTech.

[5] A. Jäderstöm,J. Matevosyan and L. Söder, "Coordinated regulation of wind power and hydro power with separate ownership", in Proc. 2005 Hydropower'05.

[6] M. Korpas, R. Hildrum and A. T. Holen, "Operation and sizing of energy storage for wind power plants in a market system", International Journal of Electrical Power and Energy Systems, No 25, pp. 599-606, Oct. 2003.

[7] E.D. Castronuovo and J.A. Pecas Lopes, "On the Optimization of the Daily Operation of Wind-Hydro Power Plant", IEEE Transactions on Power Systems, vol. 19, pp. 1599-1606, Aug. 2004.

[8] L. Söder, "A mixed rule based-linear programming applied to daily operation planning of a hydro power system", in Proc. 1995 IEEE PowerTech, pp. 93-98.

[9] O. B. Fosso, A. Gjelsvik, A. Haugstad and B. Mo, I. Wangensteen, "Generation Scheduling in a Deregulated System. Norwegian case", IEEE Transactions on Power Systems, vol. 14, pp.75-81,Feb. 1999.

[10] A. J. Conejo, "Self-Scheduling of a Hydro Producer in a Pool-Based Electricity Market", IEEE Transactions of Power Systems, vol. 17 pp. 1265-1272, Nov. 2002.

[11] H. Habibollahzadeh and J. A. Bubenko, "Applications of decomposition techniques to short-term operation planning of hydro-thermal power systems", IEEE Transactions on Power Systems, vol. PWRS-1, pp. 41-47, Feb. 1986.

[12] O. Nilsson and D. Sjelvgren, "Mixed-integer p rogramming applied to short-term planning of a hydro-thermal system", IEEE Transactions on Power Systems, vol.11, pp. 281-286, Feb. 1996.

[13] P. J. Brockwell and R. A. Davis, Time Series: Theory and Methods, (2nd ed.), Springer-Verlag, 1991.

[14] L. Söder, "Simulation of Wind Speed Forecast Errors for Operation Planning of Multi-Area Power System", in Proc. 2004 International Conference on Probability Methods Applied to Power Systems (PMAPS).

[15] J. Sveca and L. Söder, "Minimization of Imbalance Costs Bidding Wind Power on the Short Term Power Market ", in Proc. 2005 IEEE PowerTech.

[16] H. Heitsch and W. Römisch, "Scenario Reduction Algorithms in Stochastic Programming", Computational Optimization and Applications, Kluwer Academic Publishers, No. 24, pp. 187-206, 2003.

[17] J. Dupacova, N. Gröwe-Kuska and W. Römisch "Scenario Reduction in Stochastic Programming an Approach Using Probability Metrics" Mathematical Programming Ser. A 95: 2003, pp.493-511.

[18] P. Kall, S. W. Wallace, Stochastic Programming, 1st ed., John Wiley and Sons, 1994

\section{BIOGRAPHIES}

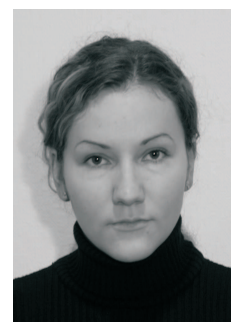

Julija Matevosyan was born in Riga, Latvia in 1978. She received her B.Sc. degree in Electrical Engineering from Riga Technical University, Latvia, in 1999; M.Sc. and Tech.Lic. degree in Electrical Engineering from the Royal Institute of Technology, Stockholm, Sweden in 2001 and 2003 resp. She is currently working towards her Ph.D. on large-scale integration of wind power in areas with limited export capability at the Royal Institute of Technology.

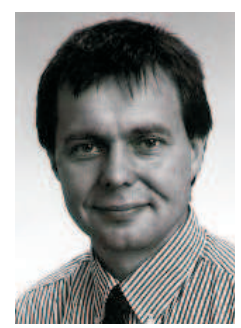

Lennart Söder (M’ 91) was born in Solna, Sweden in 1956. He received his M.Sc. and Ph.D. degrees in Electrical Engineering from the Royal Institute of Technology, Stockholm, Sweden in 1982 and 1988 respectively. He is currently a professor in Electric Power Systems at the Royal Institute of Technology. He also works with projects concerning deregulated electricity markets, distribution systems and integration of wind power. 